\title{
BMJ open Self-reported cocaine use, emergency physician testing and outcomes in suspected acute coronary syndromes: a nested matched case-control study
}

\author{
Yang Wang, ${ }^{1}$ Christopher J Lindsell, ${ }^{2}$ Charles V Pollack Jr, ${ }^{3}$ Judd Hollander, ${ }^{4}$ \\ Deborah B Diercks, ${ }^{5} \mathrm{~J}$ Douglas Kirk, ${ }^{5}$ Venkataraman Anantharaman, ${ }^{6}$ \\ W Brian Gibler, ${ }^{2}$ James Hoekstra, ${ }^{7}$ W Frank Peacock ${ }^{8}$
}

To cite: Wang Y, Lindsell CJ, Pollack CV Jr, et al. Self-reported cocaine use, emergency physician testing, and outcomes in suspected acute coronary syndromes: a nested, matched case-control study. BMJ Open 2012;2:e000572. doi:10.1136/ bmjopen-2011-000572

\section{- Prepublication history for this paper is available online. To view this file please visit the journal online (http://dx. doi.org/10.1136/ bmjopen-2011-000572).}

Received 3 November 2011 Accepted 30 March 2012

This final article is available for use under the terms of the Creative Commons Attribution Non-Commercial 2.0 Licence; see http://bmjopen.bmj.com
For numbered affiliations see end of article.

Correspondence to Dr Yang Wang; yangerwanger@gmail.com

\section{ABSTRACT}

Objectives: The primary purpose was to compare the odds of acute coronary syndrome-pertinent diagnostic testing between self-reported cocaine users and nonusers at the turn of the century. The secondary purpose was to compare the odds of acute coronary syndrome outcomes between cocaine users and non-users.

Design: Nested matched case-control study using data from the Internet Tracking Registry of Acute Coronary Syndromes.

Setting: Extracted data of patients from eight US institutions composed of six academic and two community hospitals, with census varying between 10000 and 160000 visits per year.

Participants: 249 cases of self-reported cocaine users and 249 matched controls. Matching was based on age, race, sex and any history of known coronary artery disease. Exclusion criteria were new ST elevations on initial ECG and initial physician impression of acute myocardial infarction.

\section{Primary and secondary outcome}

measures: Primary outcome was the conditional odds of undergoing non-invasive and invasive testing for coronary artery disease. Secondary outcome was the occurrences of adverse cardiac outcomes within 30 days.

Results: Cocaine users underwent diagnostic testing at similar rates compared with non-users $(9.6 \%$ vs $8.0 \%, \mathrm{OR} 1.24, \mathrm{Cl} 0.65$ to 2.34$)$. Adverse cardiovascular outcomes occurred in four $(1.6 \%)$ cocaine users and in seven $(2.8 \%)$ controls.

Conclusions: There was no increase in tendency for testing associated with self-reported history of cocaine use between 1999 and 2001. This suggests that even 10 years ago, cocaine use already had only a limited role in the Emergency Department (ED) physician's decision-making process. Similar data analyses of detailed registries can offer important contextual information that can better direct resources for future comparative effectiveness research.

\section{ARTICLE SUMMARY}

Article focus

- Was emergency physicians' tendency for noninvasive cardiac testing in chest pain patients affected by patients' self-reported history of cocaine use prior to studies over the last 10 years which have shown no benefits of noninvasive testing in the cocaine-chest pain population?

Key messages

- There was no association between patients' selfreport of cocaine use and physicians' testing tendency.

- Even prior to recent studies supporting a minimal testing strategy, emergency physicians were already keeping testing to a minimum in patients with cocaine chest pain, and earlier understanding of that practice pattern may have reduced the amount of resources spent on subsequent studies of non-invasive testing.

- Data analysis of detailed registries can be an important tool in establishing practice patterns from which further comparative effectiveness research can be more selectively conducted.

Strengths and limitations of this study

- Data are obtained from a large multicenter registry of patients with undifferentiated chest pain, which means the results are fairly representational of patients and physician practice patterns across the USA.

- The database contained a low overall prevalence of self-reported cocaine use, which means there was inadequate power to detect any statistically significant differences in morbidity/mortality.

\section{INTRODUCTION}

Cocaine is the most commonly reported illicit drug abuse among patients presenting to EDs; an estimated $5 \%-10 \%$ of the US population has used cocaine, and it is associated with more hospital visits and deaths 
than any other drug abuse. ${ }^{1}$ Among patients presenting to EDs with chest pain syndrome (CPS), $17 \%$ test positive for cocaine on urine drug screen. ${ }^{2}$ Owing to the drug's powerful sympathomimetic properties, acute cocaine intoxication has been associated with severe hypertension, coronary vasospasm, myocardial infarction and cardiac arrest. ${ }^{3-6}$ Long-term cocaine abuse has been shown to cause accelerated atherosclerosis, left ventricular hypertrophy and dilated cardiomyopathy, thus placing patients at higher risk of adverse cardiac events. ${ }^{3} 7$ While cocaine's adverse cardiac effects have been well characterised, recent studies have revealed that low-risk patients who presented to EDs with cocaineassociated CPS can be safely discharged after a $23 \mathrm{~h}$ observation period without further non-invasive testing ${ }^{8} 9$ if serial ECGs and cardiac markers were normal. In fact, over the last decade, multiple studies of various non-invasive cardiac tests have only shown that none of the tests are truly beneficial in the low-risk cocaine-related chest pain population..$^{10-13}$ These efforts have given today's ED physicians firm evidence for a streamlined approach to cocaine-associated CPS. However, whether self-reported cocaine use affected an ED physician's tendency to pursue cardiac testing prior to these more recent findings had not been well described and we questioned whether physicians were actually subjecting cocaine-related CPS patients to extensive cardiac testing. We used data from the Internet Tracking Registry of Acute Coronary Syndrome $\left(I^{*} \operatorname{tr} A C S\right)$ to compare the odds of diagnostic testing for CPS patients who reported recent cocaine use and who did not between 1999 and 2001.

\section{METHODS}

$I^{*} \operatorname{tr} A C S$ is a multicenter registry of over 17000 patients who presented to one of the eight US or one non-US ED between 1999 and 2001 with suspicion of acute coronary syndrome (ACS). Prospective data, including presenting signs and symptoms, ECG findings and the ED physician's initial impression of risk, were systematically collected. Medical record review or daily follow-up was used to obtain cardiac biomarker results, invasive and non-invasive testing, treatments, procedures and inhospital outcomes. Medical record review and telephone follow-up were used to obtain 30-day outcomes. Further details of the registry have been published previously. ${ }^{14}$

For this analysis, we extracted data for patients presenting to one of the eight institutions in the US; non-US institutions may not have similar practice patterns owing to differences in culture or care standards. The eight US institutions formed a representative cross section of providers in the USA. There were six academic and two community hospitals, with census varying between 10000 and 160000 visits during the study period. Providers of care to indigent and nonindigent populations were both well represented, with the proportion of patients receiving Medicaid or uninsured ranging from $17 \%$ to $67 \%$. Patients with new
ST-segment elevation on the presenting ECG or with an initial impression of acute myocardial infarction (AMI) were not included since management of these patients was likely independent of underlying cardiac risk factors. At the time of the registry data collection, physicians were asked to make a distinction between AMI and unstable angina/non-Q-wave myocardial infarction when making an initial impression before results of any cardiac biomarkers were obtained. From among the remaining patients, cases were selected based on a selfreported history of cocaine use and each case was then matched with a control based on 5-year age categories, race, sex and any prior history of coronary artery disease (CAD). One-to-one matching was used because selfreported cocaine use was more common among younger subjects in the registry, and there were insufficient controls for successful age matching if a higher ratio was used. Matching on additional risk factors was also not performed since the number of younger patients not reporting cocaine use included in the registry was too small.

The primary outcome was the occurrence of noninvasive or invasive assessment of CAD. Non-invasive testing was defined as exercise treadmill or rest or stress nuclear scintigraphy or echocardiography. Invasive testing was defined as percutaneous diagnostic coronary angiography. The secondary outcome was a composite outcome of confirmed ACS, coronary revascularisation or all-cause mortality within 30 days of the index ED visit. Confirmed ACS was defined as reversible ischaemia on provocative testing, CAD documented to be $>70 \%$ on coronary angiography or non-ST-segment elevation AMI as determined by positive cardiac biomarkers (CK-MB, TnI or TnT). As different sites participating in the registry used different assays for measuring cardiac biomarkers, results were recorded only as positive or negative.

Data are described using means and SDs or frequencies and percentages. Because the design involved matching cases to controls, the observations (or subjects) in the analysis were not independent. To prevent the overestimation of the OR that occurs when matching occurs in the design, conditional logistic regression was used to determine whether a report of cocaine use impacted the odds of undergoing noninvasive or invasive testing. All analyses were conducted using SPSS V.14.0 (SPSS Inc.).

\section{RESULTS}

Data for 17713 visits are available in the registry. There were 14185 visits to sites in the USA. Of those visits, 647 $(4.6 \%)$ were entirely lost to follow-up. US visits were excluded for the following reasons: 217 had undocumented age, race or sex, 587 had an initial impression of AMI and 824 had new ST-segment elevations. Of the remaining 12631, there were 249 visits (cases) in which the patient self-reported cocaine use $(2.0 \%)$. Cases were successfully matched 1:1 with visits at which cocaine use 
was not reported (controls) based on age (5-year bins), race, sex and history of CAD except for a single case; one male aged $<25$ years without a history of CAD was matched with a male aged 26 years without a history of CAD. Of the 249 cases of self-reported cocaine users, 20 $(8.0 \%)$ were entirely lost to follow-up. Of the 249 matched controls, $20(8.0 \%)$ were also lost to follow-up.

Characteristics of cases and controls are described in table 1. The proportion of tobacco users was greater among the cases than among controls $(73.1 \%$ vs $43.4 \%)$, and more cases prompted an initial physician impression of high-risk chest pain $(34.9 \%$ vs $20.1 \%)$. More controls had an initial physician impression of a non-cardiac aetiology than the cases $(32.9 \%$ vs $16.5 \%)$. Statistical testing of differences was not performed due to the matched nature of the data.

Table 2 shows the rates of testing conducted among cocaine users and controls and the conditional ORs and 95\% CIs. The OR (95\% CI) for non-invasive testing and angiography are 1.55 (0.72 to 3.30$)$ and 1.00 (0.42 to $2.40)$, respectively. Overall, the rates of non-invasive testing and angiography were similar between the selfreported cocaine users and the controls, with a combined OR (95\% CI) of 1.24 (0.65 to 2.34). Table 3 shows the incidence and ORs of various methods of noninvasive myocardial perfusion evaluation. No patient had a myocardial perfusion evaluation within 30 days following hospital discharge. The primary outcomes of combined angiography or non-invasive testing occurred in only $9.6 \%$ and $8.0 \%$ of self-reported cocaine users and controls, respectively. The numbers of non-invasive and invasive procedures cannot be summed as an individual patient could have had both types of testing performed. Also, adverse events were rare in both cases and controls (1.6\% and $2.8 \%$, respectively) with only one death overall (a control) within 30 days.

\section{DISCUSSION}

We found that patients presenting to the ED with CPS and a self-reported history of recent cocaine use, without new ST-segment elevation on the presenting ECG or an initial impression of AMI, received similar rates of objective testing for $\mathrm{CAD}$ when compared with casematched control patients without a self-reported history

\begin{tabular}{|c|c|c|}
\hline & Controls & Cases \\
\hline \multicolumn{3}{|l|}{ Demographics } \\
\hline Age in years & $39.9(9.1)$ & $39.9(9.1)$ \\
\hline Female & $70(28.1)$ & $70(28.1)$ \\
\hline Male & 179 (71.9) & 179 (71.9) \\
\hline White & $40(16.1)$ & $40(16.1)$ \\
\hline African-American & $178(71.5)$ & $178(71.5)$ \\
\hline Other & $31(12.4)$ & $31(12.4)$ \\
\hline \multicolumn{3}{|l|}{ History } \\
\hline $\begin{array}{l}\text { Family history of } \\
\text { heart disease }\end{array}$ & 77 (30.9) & $81(32.5)$ \\
\hline Current smoker & $108(43.4)$ & $182(73.1)$ \\
\hline Diabetes & $33(13.3)$ & $26(10.4)$ \\
\hline Hypertension & 83 (33.3) & 79 (31.7) \\
\hline Hyperlipidaemia & $20(8.0)$ & $15(6.0)$ \\
\hline Angina & $16(6.4)$ & 18 (7.2) \\
\hline Coronary artery disease & $23(9.2)$ & $23(9.2)$ \\
\hline Congestive heart failure & $13(5.2)$ & $10(4.0)$ \\
\hline \multicolumn{3}{|l|}{ Initial impression } \\
\hline $\begin{array}{l}\text { Unstable angina/ } \\
\text { non-Q-wave MI }\end{array}$ & $13(5.2)$ & $6(2.4)$ \\
\hline High-risk chest pain & $50(20.1)$ & $87(34.9)$ \\
\hline Low-risk chest pain & $104(41.8)$ & $115(46.2)$ \\
\hline Non-cardiac chest pain & $82(32.9)$ & $41(16.5)$ \\
\hline
\end{tabular}

of cocaine use. Our study is the first to specifically report ED physicians' testing tendency for underlying CAD in low-risk patients with self-reported cocaine use during a time period when outcome data were only just emerging. Early work had suggested that patients presenting with cocaine-related CPS are at high risk for short-term adverse outcomes. ${ }^{3}{ }^{5}$ However, more recent studies have revealed that the short-term rate of adverse events for patients with cocaine-related CPS is actually lower than those with non-cocaine-related CPS. ${ }^{8}{ }^{9}$ The entry criteria of self-reported cocaine usage is clinically important as patient history is the primary means by which emergency physicians determine what level of evaluation is necessary in patients presenting with CPS.

Our finding of a lack of difference in testing tendency may initially seem surprising owing to the amount of literature in the 1990s suggesting that cocaine usage was

Table 2 Outcomes experienced among cases and controls

\begin{tabular}{|c|c|c|c|c|}
\hline & Controls & Cases & Conditional OR $(95 \% \mathrm{Cl})$ & p Value \\
\hline Non-invasive testing & $13(5.2)$ & $19(7.6)$ & $1.55(0.72$ to 3.30$)$ & 0.261 \\
\hline Angiography & $10(4.0)$ & $10(4.0)$ & $1.00(0.42$ to 2.40$)$ & 1.000 \\
\hline Primary outcome & $20(8.0)$ & $24(9.6)$ & $1.24(0.65$ to 2.34$)$ & 0.517 \\
\hline Recurrent MI & $5(2.0)$ & $2(0.8)$ & Not done-too few outcomes & \\
\hline Percutaneous coronary intervention & $1(0.4)$ & $1(0.4)$ & & \\
\hline Coronary artery bypass graft & $1(0.4)$ & $0(0.0)$ & & \\
\hline Death & $0(0.0)$ & $1(0.4)$ & & \\
\hline Revascularation, recurrent MI or death & $7(2.8)$ & $4(1.6)$ & & \\
\hline
\end{tabular}


Table 3 Rate of each type of non-invasive testing performed during hospital stay for controls and cases

\begin{tabular}{ll}
$\begin{array}{l}\text { Controls } \\
\text { N (\%) }\end{array}$ & $\begin{array}{l}\text { Cases } \\
\text { N (\%) }\end{array}$ \\
\hline $6(2.4)$ & $4(1.6)$ \\
$3(1.2)$ & $9(3.6)$ \\
$5(2.0)$ & $9(3.6)$ \\
\hline
\end{tabular}

associated with increased risk of short-term adverse outcomes. ${ }^{61516}$ However, while cocaine was reported to induce coronary vasospasm ${ }^{41718}$ and cocaine users were being reported as having a higher risk of AMI immediately after their last use, ${ }^{6}$ Amin and Hollander had reported that the majority of at-risk patients were presenting with initial ECG changes suggestive of ACS. ${ }^{15} 1920$ Our study group was fairly young, and the majority did not have multiple traditional cardiac risk factors in their histories (table 1) or any ischaemic ECG changes. More recent work by Hermann et $a l^{21}$ has shown that in young low-risk chest pain patients without a history of cocaine use, positive non-invasive cardiac tests are primarily false positives and that there is no role for non-invasive testing in such a population. Our primary outcome shows that even a decade ago, ED physicians had already in practice extended Hermann's findings to their approach to cocaine users as well that in a low-risk population, even with the possibility of additional risk conferred by cocaine use, non-invasive cardiac testing was unnecessary and suspicion of underlying CAD was low.

While self-reported cocaine users received an evaluation similar to putatively lower risk patients without cocaine use, our secondary outcome suggests that the ED physicians' clinical decision-making process was appropriate. Despite the lack of aggressive testing, the occurrence of 30-day ACS outcomes was low (2\%-3\%, table 2) and is consistent with rates reported in more recent studies of low-risk chest pain patients where cocaine users were specifically excluded. ${ }^{22} 23$

Over the last 10 years, several groups have looked at various non-invasive methods of detecting CAD in cocaine users including dobutamine stress testing, myocardial perfusion imaging or more recently CT angiography. ${ }^{10-13}$ None of the studies has convincingly demonstrated a benefit to more testing in self-reported cocaine users. In fact, results of cardiac testing in low-risk cocaine users have been similar to those found in noncocaine users: mandatory exercise stress testing results in a low rate of positive findings ${ }^{9}$; myocardial perfusion testing does not detect any reversible ischaemia in patients without ECG changes $^{11}$ and there is limited angiographic evidence of coronary disease in patients without an abnormal ECG or elevated troponins. ${ }^{24}$ Diercks $e t a l^{25}$ found a rate of positive non-invasive test results of $17 \%$ and $14 \%$ for stimulant and cocaine users admitted to a chest pain observation unit, respectively. However, whether other factors influenced either the decision for testing or the high rate of positive results was unclear, and the high positive rate may suggest this was a high-risk population at baseline.

Our data from this registry show that as far back as 10 years ago, in an otherwise low-risk population without ischaemic ECG changes, self-reported cocaine use alone did not increase ED physicians' tendency for further cardiac testing. This practice pattern has been more recently validated by studies by Weber and Cunningham. Weber found that the 30-day events rates were similar in patients with cocaine-associated chest pain whether they received an inpatient evaluation for CAD or not. Weber's reported $1.6 \%$ rate of non-fatal MIs at 30 days is similar to our combined adverse events rate of $1.6 \%{ }^{9}$ Cunningham et $a l^{8}$ found that in 219 cocaine users with low-intermediate risk of CAD presenting to an ED with CPS, discharge after an uneventful stay in a $23 \mathrm{~h}$ observation unit resulted in no missed MIs at 1-year follow-up.

Our study has several limitations. Foremost is that we were not able to differentiate between those patients who presented immediately after cocaine use and those who merely reported a prior history of cocaine use. As the highest risk period is shortly after cocaine use, a sample of patients who presented later may have resulted in a lower complication rate than expected. Second, the $2 \%$ prevalence of cocaine use by self-report is much lower than the $17 \%$ prevalence of cocaine use confirmed by laboratory results cited by other studies. The potential lack of detection of cocaine in some percentage of the non-cocaine group may have made the two groups more similar than different. However, the rates of non-invasive testing and adverse events in both groups were already so low that any more rigorous distinction of users from non-users would probably not have been able to reduce the control group's rates to any statistically or clinically significant degree. Third, while matching was based on demographics and any known CAD, we did not match for the presences of other cardiac risk factors. Since physicians use cardiac risk factors to help determine the extent of cardiac testing, a more rigorous case matching may have eliminated several possible confounders. However, too-rigorous matching could also result in overestimation of effects, and despite the large sample size of the registry, we found that we were already not able to completely match the two groups. The only two notable differences between our cases and controls, more tobacco use and more initial impressions of high-risk chest pain in the cocaine users, would have been expected to bias our results towards a greater difference in testing tendency between the two groups. The lack of a difference in testing tendency despite the differences suggests that further matching may not be necessary and that the cases and controls were somewhat homogeneous. Fourth, it is possible that practice patterns were hospital 
dependent, so we conducted a sensitivity analysis that adjusted the model for the primary outcome for site. The conditional OR for the primary outcome in that analysis was 0.80 (95\% CI 0.39 to 1.66 ), $\mathrm{p}=0.556$, which does not change our conclusion. We note that the magnitude of the difference between cases and controls was only $1.6 \%$, and in our data, the proportion of discordant pairs was 0.15 . The observed power was therefore about $9 \%$. With a sample size of 249 pairs, the difference in proportions would need to be $6.8 \%$ or greater to have achieved statistical significance. Lastly, by specifically excluding patients with ST elevations on ECG or those with initial impressions of AMI from our study, we selected lower risk cocaine users without obvious acute pathophysiology. This was consistent with our intention to determine the impact of a self-reported history of cocaine use on emergency physicians' management strategy. While exclusion of those with obvious acute presentations may have underestimated the incidence of diagnostic testing in all cocaine users, the presence of concerning ECG changes or elevated biomarkers would have led to further cardiac testing in any patient regardless of history.

Our study is a descriptive evaluation of ED physicians' practice patterns in managing self-reported cocaine users presenting with a single episode of acute chest pain 10 years ago. Our patients were relatively young and had few risk factors for adverse cardiac events. Our analysis was not powered to detect a difference in the rate of adverse cardiac events. Our low rates at 30-day follow-up should not be interpreted as an accurate reflection of life-long cardiac disease burden in cocaine users and certainly does not reflect long-term consequences of cocaine use. Especially since others have found that even in cocaine addicts with a mean age of 32 years, $36 \%$ had $>75 \%$ atherosclerotic stenosis in at least one epicardial coronary artery. ${ }^{3}$ Also, the $1.6 \%$ recurrent MIs in Weber's study were found exclusively in those who continued to use cocaine. ${ }^{9}$ Chronic or older cocaine users probably require closer routine monitoring and may benefit from outpatient non-invasive testing, longterm follow-up and drug dependence interventions. In fact, while a history of cocaine use may not have a significant role in an ED physician's decision-making process regarding diagnostic testing, it should be noted that current ACS treatment recommendations do vary based upon recent use of cocaine, ${ }^{26}$ and therefore, it is still important to solicit this information in the ED. Future studies may be needed to further define the morbidity or mortality benefits of earlier initiation of outpatient cardiac testing in cocaine users.

Our findings are consistent with currently published guidelines on the management of cocaine chest pain and should not alter them. However, our findings do highlight the utility of registry data. During the last decade, multiple studies have been conducted on extensive testing strategies, despite the fact that a minimalist practice pattern was already in place and was yielding a very low rate of adverse outcomes. In fact, no study on non-invasive cardiac testing protocols in a similar population has demonstrated any improvement in overall mortality beyond what has been shown with a $23 \mathrm{~h}$ observation period. The $\mathrm{I} * \operatorname{tr} A C S$ registry was compiled in an era when electronic medical records (EMRs) were still under development, and data entry was done by hand. While raw data were collected between 1999 and 2001, the registry was not completed and published until 2006. The availability of computerised means of data collection and extraction would mean earlier availability of descriptive and outcome reports. If, over a decade ago, we had EMRs efficiently providing quality data to help us describe and evaluate the treatment patterns for cocaine-related chest pain patients, we may have potentially spared all the more recent resources that were used to disprove the utility of non-invasive cardiac testing. As EMRs become more advanced and ubiquitous, we have the opportunity to build detailed registries across the entire spectrum of disease processes encountered in the ED. The increased focus on comparative effectiveness research means that descriptive outcomes studies will only become more vital in establishing the contextual background against which different therapies may be compared. Without an understanding of established practice patterns and outcomes, we cannot know what, much less how, to improve upon them.

\section{CONCLUSIONS}

We found that between 1999 and 2001, in patients presenting to the ED with CPS but without ECG changes or an initial impression of AMI, there was no association between physician practice patterns and a self-reported history of cocaine use. Furthermore, the risk of ACS events within 30 days of presentation was low. Our findings show that almost 10 years prior to recent prospective studies validating the safety of a $23 \mathrm{~h}$ observation protocol and disproving the utility of extensive noninvasive cardiac testing, ED physicians were already electing for a minimally involved investigation. Furthermore, the low rate of adverse events associated with their practice pattern has yet to be significantly reduced by any more recent published studies involving more extensive cardiac testing protocols. Our study illustrates the importance of registries in patient-centred outcomes research. In the era of EMRs, the ability to efficiently build registries and generate outcomes data will be essential as focus shifts towards comparative effectiveness research and more efficient utilisation of resources.

\section{Author affiliations}

${ }^{1}$ Department of Emergency Services, David Grant Medical Center, Travis Air Force Base, California, USA

${ }^{2}$ College of Medicine, University of Cincinnati, Cincinnati, Ohio, USA

${ }^{3}$ Department of Emergency Medicine, Pennsylvania Hospital, Philadelphia, Pennsylvania, USA

${ }^{4}$ Department of Emergency Medicine, University of Pennsylvania, Philadelphia, Pennsylvania, USA 
${ }^{5}$ Department of Emergency Medicine, University of California, Davis Medical Center, Sacramento, California, USA

${ }^{6}$ Department of Emergency Medicine, Singapore General Hospital, Singapore, Singapore

${ }^{7}$ Department of Emergency Medicine, Wake Forest University Health Sciences, Winston-Salem, North Carolina, USA

${ }^{8}$ Department of Emergency Medicine, Cleveland Clinic, Cleveland, Ohio, USA

Contributors YW, CJL and WFP were involved in the conception and design, acquisition of data and analysis and interpretation of data; YW and WFP were involved in drafting the article; all authors were involved in critical revisions for important intellectual content and have given final approval of the version to be published.

Funding Although this research received no specific grant from any funding agency in the public, commercial or not-for-profit sectors, the initial I*trACS registry was supported by a grant in part by Millenium Pharmaceuticals and Schering-Plough Pharmaceuticals.

Competing interests None.

Provenance and peer review Not commissioned; externally peer reviewed.

Data sharing statement There are no additional data available for this study.

\section{REFERENCES}

1. Brody SL, Slovis CM, Wrenn KD. Cocaine-related medical problems: consecutive series of 233 patients. Am J Med 1990;88:325-31.

2. Hollander JE, Todd $\mathrm{KH}$, Green $\mathrm{G}$, et al. Chest pain associated with cocaine: an assessment of prevalence in suburban and urban emergency departments. Ann Emerg Med 1995;26:671-6.

3. Dressler FA, Malekzadeh S, Roberts WC. Quantitative analysis of amounts of coronary arterial narrowing in cocaine addicts. Am J Cardiol 1990;65:303-8.

4. Flores ED, Lange RA, Cigarroa RG, et al. Effect of cocaine on coronary artery dimensions in atherosclerotic coronary artery disease: enhanced vasoconstriction at sites of significant stenoses. J Am Coll Cardiol 1990;16:74-9.

5. Hsue PY, McManus D, Selby V, et al. Cardiac arrest in patients who smoke crack cocaine. Am J Cardiol 2007;99:822-4.

6. Mittleman MA, Mintzer D, Maclure M, et al. Triggering of myocardial infarction by cocaine. Circulation 1999;99:2737-41.

7. Wilson LD. Rapid progression of coronary artery disease in the setting of chronic cocaine abuse. J Emerg Med 1998;16:631-4.

8. Cunningham R, Walton MA, Weber JE, et al. One-year medical outcomes and emergency department recidivism after emergency department observation for cocaine-associated chest pain. Ann Emerg Med 2009;53:310-20.

9. Weber JE, Shofer FS, Larkin GL, et al. Validation of a brief observation period for patients with cocaine-associated chest pain. N Engl J Med 2003;348:510-17.

10. Dribben WH, Kirk MA, Trippi JA, et al. A pilot study to assess the safety of dobutamine stress echocardiography in the emergency department evaluation of cocaine-associated chest pain. Ann Emerg Med 2001;38:42-8.

11. Feldman JA, Bui LD, Mitchell PM, et al. The evaluation of cocaineinduced chest pain with acute myocardial perfusion imaging. Acad Emerg Med 1999;6:103-9.

12. Kontos MC, Schmidt KL, Nicholson CS, et al. Myocardial perfusion imaging with technetium-99m sestamibi in patients with cocaineassociated chest pain. Ann Emerg Med 1999;33:639-45.

13. Walsh $\mathrm{K}$, Chang AM, Perrone $\mathrm{J}$, et al. Coronary computerized tomography angiography for rapid discharge of low-risk patients with cocaine-associated chest pain. J Med Toxicol 2009;5:111-19.

14. Lindsell CJ, Anantharaman V, Diercks D, et al. The Internet Tracking Registry of Acute Coronary Syndromes (i*trACS): a multicenter registry of patients with suspicion of acute coronary syndromes reported using the standardized reporting guidelines for emergency department chest pain studies. Ann Emerg Med 2006;48:666-77, 677.e1-9.

15. Amin M, Gabelman G, Karpel J, et al. Acute myocardial infarction and chest pain syndromes after cocaine use. Am J Cardiol 1990;66:1434-7.

16. Mouhaffel AH, Madu EC, Satmary WA, et al. Cardiovascular complications of cocaine. Chest 1995;107:1426-34.

17. Lange RA, Cigarroa RG, Yancy CW Jr, et al. Cocaine-induced coronary-artery vasoconstriction. N Engl J Med 1989;321:1557-62.

18. Moliterno DJ, Willard JE, Lange RA, et al. Coronary-artery vasoconstriction induced by cocaine, cigarette smoking, or both. N Engl J Med 1994;330:454-9.

19. Hollander JE, Hoffman RS, Gennis $P$, et al. Prospective multicenter evaluation of cocaine-associated chest pain. Cocaine Associated Chest Pain (COCHPA) Study Group. Acad Emerg Med 1994:1:330-9.

20. Hollander JE, Hoffman RS, Burstein JL, et al. Cocaine-associated myocardial infarction. Mortality and complications. Cocaineassociated myocardial infarction study group. Arch Intern Med 1995; 155:1081-6.

21. Hermann LK, Weingart SD, Duvall WL, et al. The limited utility of routine cardiac stress testing in emergency department chest pain patients younger than 40 years. Ann Emerg Med 2009;54:12-16.

22. Mitchell AM, Garvey JL, Chandra A, et al. Prospective multicenter study of quantitative pretest probability assessment to exclude acute coronary syndrome for patients evaluated in emergency department chest pain units. Ann Emerg Med 2006;47:447.

23. Kline JA, Zeitouni RA, Hernandez-Nino J, et al. Randomized trial of computerized quantitative pretest probability in low-risk chest pain patients: effect on safety and resource use. Ann Emerg Med 2009;53:727-35.e1.

24. Kontos MC, Jesse RL, Tatum JL, et al. Coronary angiographic findings in patients with cocaine-associated chest pain. J Emerg Med 2003;24:9-13.

25. Diercks DB, Kirk JD, Turnipseed SD, et al. Evaluation of patients with methamphetamine- and cocaine-related chest pain in a chest pain observation unit. Crit Pathw Cardiol 2007;6:161-4.

26. McCord J, Jneid H, Hollander JE, et al. Management of cocaineassociated chest pain and myocardial infarction: a scientific statement from the American Heart Association Acute Cardiac Care Committee of the Council on Clinical Cardiology. Circulation 2008;117:1897-907. 